
\title{
25 Research Suare \\ Met Mutation is a Potential Therapeutic Target for Advanced Endometrial Cancer
}

\author{
Yu-Min Yeh \\ National Cheng Kung University Hospital

\section{Pei-Ying Wu} \\ National Cheng Kung University Hospital

\section{Peng-Chan Lin} \\ National Cheng Kung University Hospital

\section{Pei-Fang Su} \\ National Cheng Kung University

\section{Ya-Ting Hsu} \\ National Cheng Kung University Hospital \\ Jang-Yang Chang \\ National Health Research Institutes

\section{Keng-Fu Hsu} \\ National Cheng Kung University Hospital
}

Meng-Ru Shen ( $\nabla$ mrshen@mail.ncku.edu.tw)

Department of Obstetrics and Gynecology, National Cheng Kung University Hospital, College of Medicine, National Cheng Kung University, Taiwan;

\section{Research}

Keywords: endometrial cancer, MET mutation, germline variant, targeted therapy, chemoresistance

Posted Date: September 28th, 2020

DOI: https://doi.org/10.21203/rs.3.rs-80612/v1

License: (c) (i) This work is licensed under a Creative Commons Attribution 4.0 International License. Read Full License 


\section{Abstract}

\section{Background}

An optimal therapeutic regimen for endometrial cancer with extra-uterine metastasis is unavailable. This study aimed to improve our understanding about the genomic landscape of advanced endometrial cancer and identify potential therapeutic targets.

\section{Methods}

The clinical and genomic profiles of 81 patients with stage III or IV endometrial cancer were integrated. The genomic landscape was compared with that of The Cancer Genome Atlas (TCGA) cohort. To identify genomic aberrations associated with clinical outcome, Cox proportional hazard regression was used. The impacts of the genomic aberrations were validated in vitro and in vivo.

\section{Results}

A discrepancy in major genetic aberrations that contributed to the genomic functions was observed between the present study and TCGA cohorts. The mutation status of MET, U2AF1, BCL9, PPP2R1A, IDH2, $C B L, B T K$, and $C H E K 2$ were positively correlated with poor clinical outcomes. MET mutations occurred in $30 \%$ of the patients who presented with poor overall survival (hazard ratio, 2.606; $95 \%$ confidence interval, 1.167 5.819; adjusted p-value, 0.067). Concurrent MET and KRAS mutations presented with the worst outcomes. MET mutations in HGF-binding $(58.1 \%)$ or kinase $(16.2 \%)$ domains resulted in differential HGF-induced c-MET phosphorylation. Different types of MET mutations differentially affected tumor growth and displayed different sensitivities to cisplatin and tyrosine kinase inhibitors. MET N375S mutation is a germline variant with a high incidence in Eastern Asia and causes chemoresistance to cisplatin.

\section{Conclusions}

This study highlights the ethnic differences in the biology of the disease, which can influence the treatment recommendations and the genome-guided clinical trials of advanced endometrial cancer.

\section{Background}

Endometrial cancer, the most common gynecological cancer in developed countries, is broadly classified into two types (type I and type II), based on its histology and the presence or absence of a hormone receptor. ${ }^{1}$ Type I endometrioid cancer is associated with estrogen excess, obesity, hormone-receptor positivity, and a favorable prognosis, as compared to type II endometrial cancers, which present as serous tumors that are more common in non-obese women and exhibit a poorer outcome. ${ }^{2}$ The 5 -year overall survival rate ranges from $74-91 \%$ at the early stage (International Federation of Gynecology and Obstetrics, FIGO stages I or II). In contrast, the 5-year overall survival rates in patients with extra-uterine metastatic disease are $57-66 \%$ and $20-26 \%$ for FIGO stages III and IV, respectively. ${ }^{3}$ Extra-uterine spread 
is observed in about $25 \%$ of patients who are newly diagnosed with endometrial, and chemotherapy is the suggested mode of treatment. However, the optimal treatment of this cancer has not been determined so far. In general, platinum-based chemotherapy was used as the first-line treatment for metastatic or advanced endometrial cancer. However, no standard protocol for the second-line option when the tumor persists or recurs exists. ${ }^{4}$

The Cancer Genome Atlas (TCGA) Research Network produced a vast amount of data on the genomic landscape of endometrial cancer. ${ }^{5}$ The genomic analysis categorized endometrial cancer into subgroups based on distinct molecular characteristics: the group with DNA polymerase epsilon (POLE) somatic mutations and the corresponding 'ultramutated' phenotype exhibit a favorable prognosis, while the other distinct subgroups, which include tumors with microsatellite instability, low copy number, and high copy number, consisted mostly of cases diagnosed as high-grade serous tumors with poor outcome. The treatment for metastatic disease is not well established, and the survival of patients with this disease has not improved over the last decade. No targeted therapy, except for hormonal therapy, has been approved so far. ${ }^{6}$ Therefore, the identification of the biological pathways that may be altered in advanced endometrial cancer might aid in improving the clinical management of this disease.

This study aimed to identify the survival-associated molecular pathways in advanced endometrial cancer via integrated genomic characterization.

\section{Methods}

Study cohort. Patients with endometrial cancer were consecutively recruited the National Cheng Kung University Hospital Taiwan from July 2006 to January 2017. About 900 patients with endometrial cancer were enrolled, and $20 \%$ of cases were diagnosed with advanced-stage cancer (FIGO stage III or IV). The patients received staging surgery or diagnostic dilation and curettage at initial diagnosis. Platinum plus paclitaxel were the standard regimens used as post-operative chemotherapy for patients with FIGO stage III and IV. The patients were recruited as an NCKUH cohort using the following inclusion criteria: (i) FIGO stage III or IV; (ii) surgery performed at NCKUH; and (iii) adequate and qualified specimens for genetic analysis. The exclusion criteria were as follows: (i) unavailable clinical information; (ii) death unrelated to cancer; and (iii) poor quality of specimens. This study was approved by the institutional review board of NCKUH (A-ER-103-151, A-ER-103-395, and A-ER-104-153). Whole-genome sequencing (WGS) data of 499 normal Taiwanese individuals were provided by the Taiwan Biobank as genome reference.

Next-generation sequencing. Genomic analysis was performed on formalin-fixed paraffin-embedded (FFPE) tumor tissues using Oncomine Comprehensive Assay ${ }^{\mathrm{TM}} \mathrm{v}$ 1 (ThermoFisher, MA, USA). ${ }^{7}$ Hematoxylin and eosin (H\&E)-stained FFPE sections were reviewed to ensure that the tumor content was $>50 \%$. Genomic DNA and RNA were extracted from the sections using the Recover All Total Nucleic Acid Isolation Kit (Thermo Fisher Scientific). RNA was reverse-transcribed to produce cDNA using the SuperScript VILO cDNA Synthesis Kit (Thermo Fisher Scientific). Target regions from genomic DNA and cDNA were amplified using the DNA Oncomine Cancer Research and RNA Oncomine Cancer Research 
panels (Thermo Fisher Scientific). Library construction of the amplicons was performed according to the manufacturer's instructions using the lon AmpliSeq Library Kit 2.0 (Thermo Fisher Scientific). The template was prepared with the Ion PGM Template OT2 200 Kit (Thermo Fisher Scientific), and the Ion 318 chip (Thermo Fisher Scientific) was prepared and loaded according to the manufacturer's recommendations. The lon PGM Sequencing 200 Kit v.2 (Thermo Fisher Scientific) was used with the lon PGM sequencer (Thermo Fisher Scientific) as described in the User Guide (average sequencing depth, 1000x).

Next, POLE gene panels were designed and ordered at AmpliSeq.com to identify the pathogenic mutations in the gene. POLE gene libraries were prepared using the lon AmpliSeq ${ }^{\text {TM }}$ Kit for Chef DL8 with the lon Chef ${ }^{\mathrm{TM}}$ System. Template preparation, chip loading, and sequencing were carried out on the lon Chef $^{\mathrm{T} M}$ System and lon S5 XL sequencing system using the lon 510 \& Ion 520 \& Ion 530 Kit-Chef Kit (Thermo Fisher Scientific). The sequencing reads were aligned to the reference genome (hg19), and variant calling and annotation were conducted using lon Reporter Version 5.6.

To detect the germline genetic variant, DNA was isolated from the peripheral blood of 20 patients and adjacent non-tumor specimens of 15 patients. WGS and whole exome sequencing (WES) of the blood and tissue samples were performed using the Illumina HiSeq ${ }^{\circledR} 2500$ and lon Torren $^{\mathrm{Tm}}$ systems, $^{2}$ respectively. NGS library preparation was carried out using the TruSeq PCR-Free DNA HT Library Prep kit (IIlumina Inc). The size and concentration of the DNA library were measured using Agilent 2100 Bioanalyzer (Agilent) and Qubit Fluorometer (Life Technologies). WGS and WES were performed with a minimum median coverage of $30 \times$ and $100 \times$, respectively. FastQC was used to check the quality of the reads, which were aligned to the hg19 reference genome using the BWA-MEM algorithm. The GATK Best Practices was used for base quality score recalibration, small insertion and deletion (INDEL) realignment, and duplicate removal. ${ }^{8}$ Single nucleotide variant (SNV) and INDEL discovery and genotyping were performed according to GATK. ${ }^{9,10}$ Manta and Canvas were used for SNV and CNV discovery. ${ }^{11,12}$ To study the possible functional impacts of the associated variants, the Ensemble Variant Effect Predictor was used. ${ }^{13}$

Sitedirected mutagenesis of MET. The pCMV6-MET mutant (N375S) plasmid was purchased from ORIGENE (\#RC400336). The construction of mutated derivatives of $c-M E T$ was performed using the QuikChange site-directed mutagenesis kit (Agilent Technologies). Mutations for target nucleotide were introduced using the designed oligonucleotides and pCMV6-MET (ORIGENE; \#RC217003) as a template. The PCR products were added to Dpnl enzyme for $5 \mathrm{~min}$ at $37^{\circ} \mathrm{C}$ to destroy the parental plasmid DNA and then transformed into $E$. coli. All mutated constructs were confirmed by sequencing.

Cell culture and functional assays. In April 2017, authenticated endometrial cancer cell lines, RL95-2 and $K L E$, were purchased from the American Type Culture Collection. Cell functions on proliferation, migration, and invasion were performed as previously described ${ }^{14}$. Briefly, the RL95-2 and KLE cell lines transfected with wild-type and mutant $c$-MET were seeded into 96-well plates $\left(5 \times 10^{3} /\right.$ well) with $100 \mu$ l of culture medium and incubated at $37^{\circ} \mathrm{C}$ with $5 \% \mathrm{CO}_{2}$. Cell proliferation was evaluated $0,24,48$, and $72 \mathrm{~h}$ after 
seeding using the Alamar Blue assay (Invitrogen). Next, transwell assay was used to evaluate the migration and invasion abilities of the transfected cells. For the invasion assay, $100 \mu \mathrm{l}$ of matrigel (10\%; BD Biosciences) was added to the bottom of the transwell insert. Next, $3 \times 10^{4}$ transfected cells were seeded on to the transwell insert along with $300 \mu \mathrm{l}$ of medium containing $5 \% \mathrm{FBS} ; 1 \mathrm{ml}$ of medium with $10 \%$ FBS was added to the lower part of the 24 -well plate. The cells were allowed to invade or migrate for $24 \mathrm{~h}$ at $37^{\circ} \mathrm{C}$ with $5 \% \mathrm{CO}_{2}$. All the cells from the underside of the transwell inserts were stained by crystal violet. Images of each transwell insert were taken to count the number of cells.

c-MET phosphorylation. The cells were washed with phosphate-buffered saline (PBS) and lysed in cell lysis buffer (10 mM Tris pH7.4, $150 \mathrm{mM} \mathrm{NaCl}, 1 \mathrm{mM}$ ethylenediaminetetraacetic acid [EDTA], 1\% octylphenoxypolyethoxyethanol (IGEPAL), $0.5 \%$ deoxycholic acid, and $0.1 \%$ SDS) containing freshly added Protease Inhibitor Cocktail (Sigma, St Louis, MO, USA) and $1 \mathrm{mM}$ phenylmethanesulfonylfluoride (PMSF). The protein concentration of the cell lysate was determined using the DC Protein Assay (Bio-Rad Laboratories, Hercules, CA, USA). Equal amounts of lysate protein $(30 \mu \mathrm{g})$ from each cell lysate were loaded on to $4-15 \%$ SDS-polyacrylamide gel (Bio-Rad) and separated by electrophoresis. The separated proteins were electroblotted onto a nitrocellulose membrane $(0.45 \mu \mathrm{m}$; Bio-Rad) and incubated in blocking solution (1X PBS, $0.1 \%$ Tween-20, 5\% non-fat dry milk powder) for $1 \mathrm{~h}$ at room temperature. The membranes were incubated with the following dilutions of the primary antibody at $40{ }^{\circ} \mathrm{C}$ overnight: antiphospho-c-MET, 1:1,000 (\#3077; Cell Signaling Technology); anti-c-MET, 1:2,000 (\#8198; Cell Signaling Technology); and anti-a-tubulin, 1:5,000 (DM1A; Novus Biologicals). After multiple washes with PBS containing $0.1 \%$ Tween-20 (PBST), the membranes were incubated with horseradish peroxidaseconjugated secondary antibody (goat anti-mouse or goat anti-rabbit IgG; Bio-Rad) for $1 \mathrm{~h}$ at room temperature. After further washes with PBST, the membranes were processed using the enhanced chemiluminescence method (SuperSignal West Pico substrate; Pierce, Rockford, IL, USA). Protein bands were visualized by autoradiography, and the signal intensities were quantified by using the NIH ImageJ software.

Immunohistochemical staining. FFPE endometrial cancer tissues from surgical specimens were used to determine the expression of c-MET protein. Tissue sections were cut from the paraffin block (thickness, $4 \mu \mathrm{m})$, deparaffinized in xylene, and rehydrated with decreasing grades of ethanol. Immunohistochemical staining for the MET protein was performed using the anti-MET rabbit polyclonal antibody (sc-161; Santa Cruz Biotechnology). The expression level of MET in tumors with wild-type $c-M E T$ was compared with those in tumors carrying various $c-M E T$ mutations.

In silico analysis. To predict the effect of variants on the function of the c-MET protein, differences in free energy between mutant and wild-type MET were calculated using Calculate Mutation Energy. ${ }^{24}$ For the variants located in the tyrosine kinase domain, the analysis was performed using the crystal structure of the kinase domain in complex with ATP. Regarding the variants located in the Sema domain, the binding with HGF was used for estimation. Variants with mutation energy higher than $0.5 \mathrm{kcal} / \mathrm{mol}$ were reported as destabilized. 
Animal Study. All animal experiments were approved by the Laboratory Animal Care and Use Committee of the NCKU. The xenograft procedures were performed in 6-8-week-old, female, severe combined

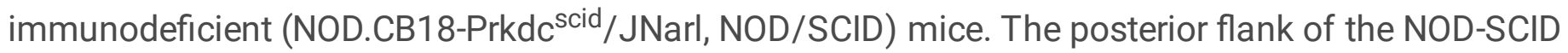
mouse was subcutaneously inoculated with $1 \times 10^{6}$ endometrial cancer RL95-2 cells expressing either wild-type or mutant MET (N375S, G1085R, G1087E). The mice were randomly divided into four groups (at least four mice in each group) and treated with cisplatin, crizotinib (multiple tyrosine kinase inhibitor), SU11274 (a specific c-MET inhibitor), or none of the aforementioned drugs; the treatments were initiated 1 week after tumor inoculation. Cisplatin $(1 \mathrm{mg} / \mathrm{kg})$ and SU11274 $(6 \mathrm{mg} / \mathrm{kg})$ were administered twice a week via intraperitoneal (i.p.) injection for 5 weeks. Crizotinib $(25 \mathrm{mg} / \mathrm{kg})$, prepared with $0.5 \%$ hydroxypropylmethylcellulose, was administered by oral gavage twice a week for 5 weeks. Both tumor size and body weight were measured twice a week, and tumor volume was determined via caliper measurements of the tumor length $(L)$ and width $(W)$ according to the following formula: $L W^{2} / 2$. The mice were sacrificed at day 40 , and the tumors were excised and weighed.

Statistics. Chi-Square test, Fisher's exact test, and unpaired $t$-test were used to compare the differences between the groups. After adjusting for the stage of the tumor, the association between individual mutated gene and survival outcome was assessed using the Cox proportional hazards regression model. The correction for multiple comparisons was performed using the False Discovery Rate (FDR) method. The cutoff for the FDR adjusted $p$-value was 0.1. Kaplan-Meier curves and log-rank tests were used to estimate the survival functions and compare the differences between groups. A p-value of $<0.05$ was considered statistically significant.

\section{Results}

Patient characteristics. Eighty-one patients with stage III and IV cancer were consecutively recruited based on the inclusion criteria. The clinical characteristics of the patients were shown in Supplementary Table S1. Based on the histology, $80 \%$ and $20 \%$ of the patients presented with Type I (mainly endometrioid) and Type II (non-endometrioid) tumors, respectively. Furthermore, the patients were grouped based on the clinical outcome as follows (Table 1): no evidence of disease and progressive disease (PD). The clinical characteristics of the patients in the two groups are shown in Table 1. No significant differences in histology, grading, and treatment were observed between the two groups of patients. 
Table 1

Patient characteristics

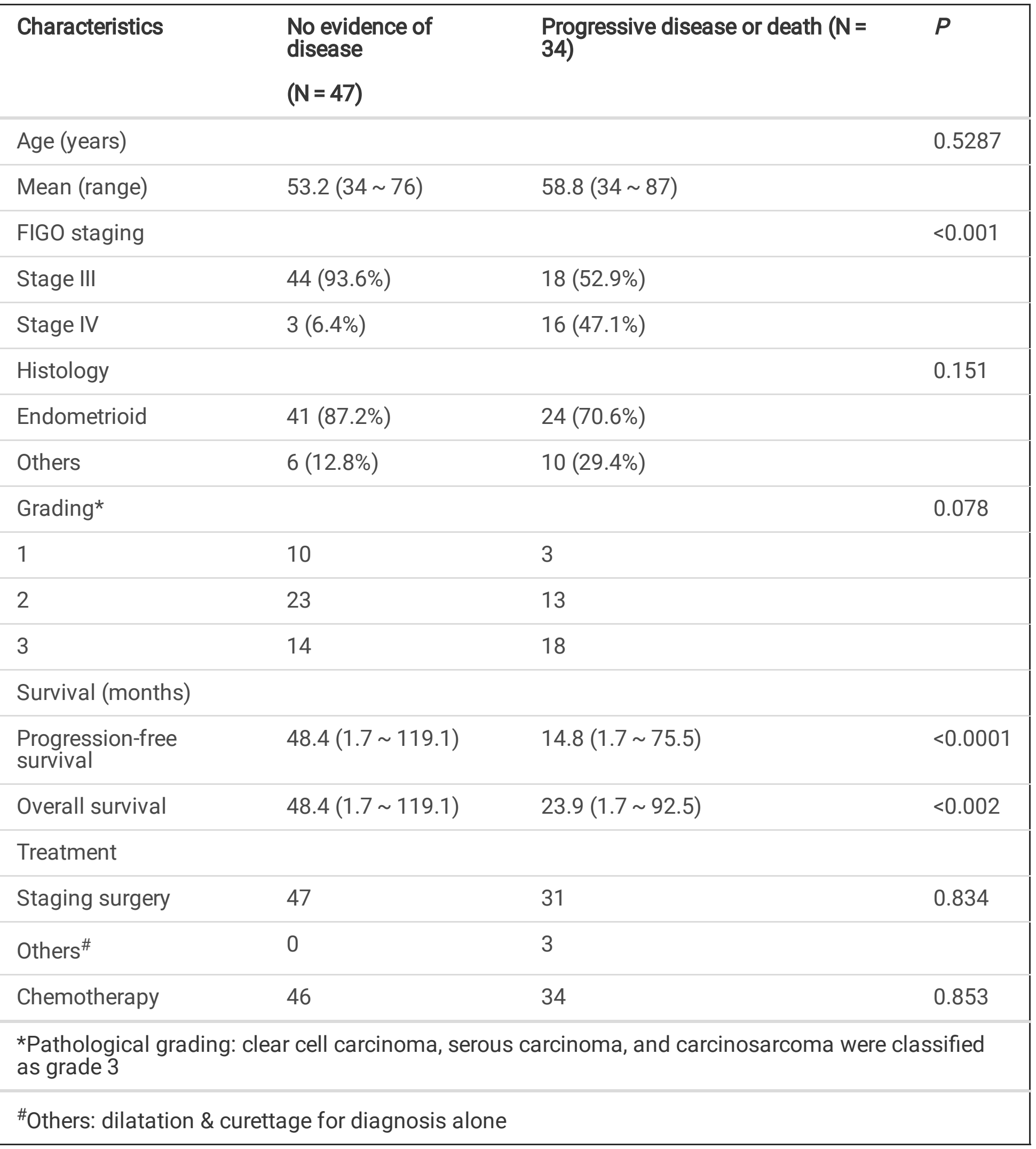

Clinical impacts of gene mutation. The Oncomine Comprehensive Assay v1, a targeted NGS assay, was applied to the FFPE tumor samples to detect the presence of mutations across 143 genes. Associations between the individual mutated gene and the clinical outcome with FDR adjusted $p$-value $<0.1$ are shown 
in Tables 2 and 3. The mutation status of eight genes (MET, U2AF1, BCL9, PPP2R1A, IDH2, CBL, BTK, and CHEK2) were positively correlated with poor progression-free survival (PFS) and overall survival (OS). In contrast, IFITM1 and DNMT3A mutations were associated with better clinical outcomes. MET was selected for further studies because the adjusted $p$-value was the smallest and the frequency of mutation was not low.

Table 2

The association between the mutated genes and overall survival.

\begin{tabular}{|lllllll|}
\hline Gene & $\begin{array}{l}\text { Case number } \\
\text { with mutation }\end{array}$ & $\begin{array}{l}\text { Case number } \\
\text { without mutation }\end{array}$ & $\begin{array}{l}\text { p value } \\
\text { valjusted }\end{array}$ & $\begin{array}{l}\text { Hazard } \\
\text { ratio }\end{array}$ & $\begin{array}{l}\text { 95\% interval } \\
\text { (Lower } ~ \\
\text { Upper) }\end{array}$
\end{tabular}


Table 3

The association between the mutated genes and progression-free survival.

\begin{tabular}{|c|c|c|c|c|c|c|}
\hline Gene & $\begin{array}{l}\text { Case number } \\
\text { with mutation }\end{array}$ & $\begin{array}{l}\text { Case number } \\
\text { without mutation }\end{array}$ & $\begin{array}{l}\mathrm{p} \\
\text { value }\end{array}$ & $\begin{array}{l}\text { Adjusted } \\
\mathrm{p} \text { value }\end{array}$ & $\begin{array}{l}\text { Hazard } \\
\text { ratio }\end{array}$ & $\begin{array}{l}95 \% \text { interval } \\
\text { (Lower } \\
\text { Upper) }\end{array}$ \\
\hline MET & 24 & 57 & 0.044 & 0.067 & 2.081 & $1.020 \sim 4.248$ \\
\hline BCL9 & 4 & 77 & 0.059 & 0.067 & 3.274 & $0.958 \sim 11.194$ \\
\hline TSC2 & 27 & 54 & 0.029 & 0.067 & 2.122 & $1.079 \sim 4.177$ \\
\hline NF1 & 48 & 33 & 0.052 & 0.067 & 2.222 & $0.993 \sim 4.974$ \\
\hline MAP2K2 & 2 & 79 & 0.063 & 0.067 & 4.066 & $0.926 \sim 17.854$ \\
\hline NFE2L2 & 6 & 75 & 0.062 & 0.067 & 0.244 & $0.056 \sim 1.073$ \\
\hline U2AF1 & 3 & 78 & 0.008 & 0.067 & 5.395 & $1.563 \sim 18.615$ \\
\hline CHEK2 & 4 & 77 & 0.049 & 0.067 & 3.503 & $1.007 \sim 12.184$ \\
\hline VHL & 8 & 73 & 0.020 & 0.067 & 3.293 & $1.206 \sim 8.993$ \\
\hline CBL & 2 & 79 & 0.016 & 0.067 & 6.589 & $1.425 \sim 30.475$ \\
\hline IFITM1 & 79 & 2 & 0.023 & 0.067 & 0.168 & $0.036 \sim 0.778$ \\
\hline IDH2 & 2 & 79 & 0.049 & 0.067 & 4.398 & $1.005 \sim 19.250$ \\
\hline DNMT3A & 9 & 72 & 0.036 & 0.067 & 0.267 & $0.078 \sim 0.918$ \\
\hline BTK & 2 & 79 & 0.016 & 0.067 & 6.589 & $1.425 \sim 30.475$ \\
\hline PPP2R1A & 8 & 73 & 0.087 & 0.087 & 2.329 & $0.883 \sim 6.143$ \\
\hline
\end{tabular}

MET mutation is a cancer driver. MET mutation is a poor clinical marker (Fig. 1A). c-MET, a protein encoded by the human MET gene, is a receptor tyrosine kinase (RTK) expressed on the cell surface (1). The aberrant activation of the c-MET pathway and crosstalk with other RTKs has been shown to stimulate the PI3K/AKT and RAS/MAPK signaling pathways, which contribute to cancer biology (Supplementary Fig. 1A). MET mutation was associated with poor survival independent of EGFR mutation (Supplementary Fig. 1B). Alternatively, EGFR mutation did not affect the clinical outcome in patients harboring the wild-type or mutant $M E T$. Similar results were observed in the MET and ERBB2, $M E T$ and $P I K 3 R 1$, and MET and PIK3CA combination mutations (Supplementary Fig. 1C, Figs. $1 \mathrm{~B}$ and 1C). However, the impacts of KDR and KRAS mutations were different. The presence of KDR or KRAS mutations indicated poor outcomes in patients with $M E T$ mutations, but not in those without $M E T$ mutations (Supplementary Fig. 1D and Fig. 1D). Taken together, MET mutation highly influences the clinical outcome of advanced endometrial cancer, and KDR and KRAS mutations exhibit additional impacts on patients with $M E T$ mutation. 
In silico analysis of MET mutation. The types of MET mutations were analyzed in 112 samples, including 81 primary tumors and 31 metastatic tissues. A total of 35 nonsynonymous mutations, including missense mutations and small INDELs, were identified in exons 2, 10,11, 14, 15, and 16 (Fig. 2A, Supplementary Figure S2A). Aberrant MET mutations resulted in C-MET overexpression in the endometrial cancer tissues (Fig. 2B), similar to that seen in lung cancer, indicating that C-MET overexpression alone can induce oncogenic transformation in vitro and in vivo. ${ }^{16,17,18,19}$ Moreover, patients with intracellular domain mutations seemed to exhibit a worse outcome (Supplementary Figure S2B).

To predict the impact of the MET mutations on the protein structure, the "in silico" analysis was performed. ${ }^{20}$ When the interaction between the semaphorin domain of c-MET and HGF was analyzed (Supplementary Figure S3A), the exon 2 mutations in did not cause any obvious change in the predicted free energy, implying that these mutations did not theoretically affect the binding of HGF to C-MET (Supplementary Figure S3B). For mutations in the kinase domain, the free energy changes were studied in the presence of ATP (Supplementary Figure S3C). As shown in Supplementary Figure S3D, the "in silico" models demonstrated a Gly-to-Arg or Gly-to-Glu change at codon 1085 or a Gly-to-Glu change at codon 1087, which increased the mutational free energy. These findings indicated that these mutations might affect ATP binding, c-MET phosphorylation, and subsequent biological functions.

Effect of MET mutation on cellular function. To confirm the "in silico" analysis, we established various clones of MET mutants in the RL95-2 and KLE cell lines. The time course of C-MET phosphorylation was studied in response to HGF stimulation. In the wild-type c-MET RL95-2 cells, HGF induced a rapid increase in c-MET phosphorylation within $3 \mathrm{~min}$, which was gradually decreased over $120 \mathrm{~min}$ (Fig. 2C). In contrast, an equivalent increase in C-MET phosphorylation was observed, which was sustained for 120 min in exons 15 (G1085E) or 16 (G1087E) in the clones of the kinase domain mutants. In the mutated semaphorin domain N375S clones, a delayed peak in c-MET phosphorylation was noted at $30 \mathrm{~min}$, followed by rapid dephosphorylation. These results imply that different $M E T$ aberrations might exert differential effects on cellular functions. This hypothesis was confirmed by the proliferation, migration, and invasion assays in the endometrial cancer cell lines (Fig. 2D \& 2E, Supplementary Figure S4).

Endometrial cancer growth in vivo. To test whether the c-MET signaling pathway was a therapeutic target, we subcutaneously inoculated the SCID mice with RL95-2 cells carrying various MET mutations. Tumors harboring different $M E T$ mutants showed a significant increase in volume when compared to those with wild-type MET (Fig. 3A-B, Supplementary Figure S5). Next, cisplatin significantly inhibited the growth of tumors carrying the wild-type MET and the G1085R and G1087E mutations (Fig. 3C-D). However, it appeared to exhibit no inhibitory effect on tumors with MET N375S. Crizotinib, a multi-target tyrosine kinase inhibitor, significantly inhibited the growth of tumors carrying the N375S, G1085R or G1087E mutant, when compared to those with the wild-type MET (Fig. 3E-F). SU11274, a specific c-MET inhibitor, also showed potent inhibitory effects on the growth of tumors carrying G1085R or 1087E mutations, whereas its inhibitory effect on the growth of tumors carrying wild-type MET was not very obvious. Interestingly, tumors with N375S mutation were insensitive to SU11274 in vivo (Fig. 3G-H). These data 
indicate that MET mutations promote the growth of endometrial tumors and show different sensitivities to cisplatin or tyrosine kinase inhibitors (crizotinib and SU11274), depending on the type of MET mutation.

MET N375S is a germline variant. In animal studies, mutation of the semaphorin domain N375S displayed resistance to cisplatin, the major chemotherapeutic agent for endometrial cancer (Fig. $3 \mathrm{C}$ and D). In our cohort, the OS rate for patients without MET mutations was $75 \%$; however, that value significantly decreased to $50 \%$ in patients with MET N375S (Fig. $4 \mathrm{~A}, \mathrm{P}=0.043$ ). Furthermore, an analysis of 35 paired tissues revealed that the MET N375S mutation was a germline variant (Fig. 4B), consistent with the findings of a previous study in lung cancer. ${ }^{21}$ When compared to other nonsynonymous variants, the incidence of the N375S variant (c.1124 A $>$ G) was relatively high $(11 \sim 17 \%)$ in the current study and the Taiwan Biobank (Fig. 4C). The worldwide distribution of genetic N375S variants (c.1124 A>G) was assessed in the data from the 1000 Genomes Project. The allele frequency of the MET N375S variant (c.1124 A>G) was $8.5 \%$ and $5.8 \%$ in all the patients enrolled in the present study and the Taiwan Biobank, respectively. A similar allele frequency (5\% 8\%) was observed in the population from South and East Asia (Fig. 4D). In contrast, less than $2 \%$ of the alternative allele frequency was observed in the European, American, and African populations.

Comparison with TCGA molecular classification. Patients with advanced disease (stage III or IV) in the TCGA endometrial cancer cohort were identified, and the genomic backgrounds between the NCKUH and TCGA cohort were compared. No significant differences in age distribution at initial diagnosis, FIGO stage, histology, and clinical outcome were observed between the two cohorts (Supplementary Figure S6A and S6B). To obtain somatic mutations, the variants identified by Oncomine Comprehensive Assay v1 were filtered by the germline genetic variants. The top gene aberrations with high impact on genomic functions were listed after analyzing and filtering out the mutations, which were categorized as "low" (harmless or unlikely to change protein behavior), "modifier" (affecting the non-coding), or "moderate" (inframe insertion or protein-altering variant; Supplementary Figure S6C). The ranking of the top 10 genetic aberrations was different between the two cohorts, although PTEN was the most common mutated gene. Mutations in $B R A F, J A K 1$, and $K I T$ were identified in approximately $4-11 \%$ of the patients in TCGA cohort, but they were rarely detected in the NCKUH cohort. In addition, at the advanced stage, MET mutations were observed in $15 \%$ of cases in the TCGA cohort without an impact on the OS (Supplementary Figure S6D).

A subset of endometrioid cancer was newly identified in hotspot POLE mutations in the TCGA cohort. ${ }^{5}$ TCGA and subsequent studies showed that POLE-mutant endometrial cancers typically present as highgrade or poorly-differentiated tumors. ${ }^{5,22}$ In addition, POLE mutations accompanied by an ultra-tumor mutation burden present with favorable clinical outcomes. ${ }^{5,23}$ Among the 81 patients recruited in this study, 74 presented with adequate and qualified samples to investigate the pathogenic mutations in the POLE gene. However, no association between the POLE mutation and the histology of the specimens was observed in the current study cohort (Supplementary Figure S7A). In addition, the OS was not affected by 
the presence of the POLE mutation (Supplementary Figure S7B). A discrepancy in the genetic aberrations between the NCKUH and TCGA cohorts was noted.

\section{Discussion}

This study highlights the survival-associated molecular pathways in advanced endometrial cancer. MET mutation was found to be a potential therapeutic target. This conclusion was reached based on several important findings. First, the MET mutation was noted in $30 \%$ of the advanced endometrial cancer cases and was associated with a poor clinical outcome; concurrent MET and KRAS mutations indicated the worst outcome. Second, MET mutation promoted the growth and invasion of endometrial tumors both in vivo and in vitro. Third, MET mutation occurred in the HGF-binding or kinase domain. Mutations in the kinase domain induce sustained c-MET phosphorylation in response to HGF stimulation. Consistently, patients with kinase domain mutations presented with the worst clinical outcome compared to those with mutations in extracellular regions. Fourth, tumors harboring c-MET kinase domain mutations in the SCID mouse model were sensitive to the tyrosine kinase inhibitors and cisplatin. Finally, mutation of the Sema domain N375S provided resistance against cisplatin, and this effect was not overcome by the selective cMET inhibitor.

The HGF-MET signaling pathway regulates cell proliferation and motility. ${ }^{16,17}$ The Sema domain of cMET is necessary for HGF-binding, receptor dimerization, and the activation of the downstream signaling. In the present study, $58 \%$ of the MET mutations were identified in the Sema domain, and N375S was the most frequent mutation within this region. An analysis of the paired genomic DNA in normal and tumor tissues and in the data obtained from the 1000 Genome Projects revealed that MET N375S mutation was a germline variant with high frequency in the Asian population. This ethnic difference was previously reported in patients with lung cancer, and MET N375S was reported to confer resistance to c-MET inhibition. ${ }^{21}$ Earlier studies suggested that the resistance to c-MET inhibitor was caused by a missense change in the 375 serine residue, leading to a weakening in the interaction between HGF and c-MET and a decrease in kinase activation, thereby resulting in increased resistance to the c-MET inhibitor. ${ }^{24,25}$ This notion was supported by our experiments in the endometrial cancer cell line, which showed that HGF induced delayed c-MET phosphorylation accompanied by rapid dephosphorylation. In the SCID mouse models, tumors harboring MET N375S were consistently resistant to the specific c-MET inhibitor, SU11274.

Interestingly, MET N375S provided drug resistance to cisplatin in the mouse model, in the current study. Cisplatin is one of the most active chemotherapeutic agents used in endometrial cancer. The poor clinical outcome in patients with MET N375S mutation in the NCKUH cohort supported this important finding in vivo. To the best of our knowledge, this correlation with chemoresistance has not been reported so far. Given the nature of the germline genetic variant, N375S would be a "de novo" mechanism of cisplatin resistance. The selection of patients based on the germline MET genetic variant may prove more beneficial for patients with endometrial cancer receiving cisplatin-based therapy and could be a new therapeutic strategy in precision medicine.

Page $12 / 21$ 
A discrepancy in the genetic aberration, in terms of the severity of the effect of the mutation on genomic functions, was observed between the current study cohort and TCGA cohort. In TCGA cohort, POLEmutant endometrial cancers were typically high-grade or poorly-differentiated. ${ }^{5}$ POLE mutations accompanied by ultra-tumor mutation burden presented with a favorable clinical outcome. ${ }^{23}$ Conversely, in our cohort, the results showed no associations between POLE mutation, histologic subtype, and clinical outcome.

\section{Conclusions}

This integrated genomic and mechanistic study provides insights into the biology and diagnostic classification of advanced endometrial cancer, which might result in a direct effect on the treatment recommendations for patients with this disease. Furthermore, this information provides opportunities for additional genome-guided clinical trials and drug development. Since several c-MET inhibitors are FDAapproved drugs and in the late phases of the clinical trials, the next step is to launch a clinical trial intended at targeting MET mutations in advanced endometrial cancers.

\section{Abbreviations}

BWA-MEM

Burrows-Wheeler Aligner-Maximal Exact Matches

CMV

Cytomegalovirus

CNV

Copy Number Variation

DNA

Deoxyribonucleic Acid

EDTA

Ethylenediaminetetra-Acetic Acid

FDR

False Discovery Rate

FIGO

The International Federation of Gynecology and Obstetrics

GATK

Genome Analysis Tool Kit

INDEL

Insertion and Deletion

MAPK

Mitogen-Activated Protein Kinase

$\mathrm{NIH}$

National Institutes of Health 
OS

Overall Survival

PBS

Phosphate-Buffered Saline

PCR

Polymerase Chain Reaction

PFS

Progression-Free Survival

PMSF

Phenylmethanesulfonylfluoride

POLE

DNA Polymerase Epsilon

RNA

Ribonucleic Acid

SCID

Severe Combined Immunodeficient

SDS

Sodium Dodecyl Sulfate

SNV

Single Nucleotide Variant

TCGA

The Cancer Genome Atlas

WES

Whole Exome Sequencing

WGS

Whole-Genome Sequencing

FFPE

Formalin-Fixed Paraffin-Embedded

\section{Declarations}

\section{Ethics approval and consent to participate}

This clinical study was approved by the institutional review board (IRB) of NCKUH (A-ER-103-151, A-ER103-395, and A-ER-104-153) and all participants provided written informed consent. Animal studies were approved by the Institutional Laboratory Animal Care and Use Committee (IACUC) of the NCKU.

\section{Consent for publication}

Not applicable. 
The datasets used and/or analyzed during the current study are available from the corresponding author on reasonable request.

\section{Competing interests}

The authors declare that they have no competing interests.

\section{Funding}

This study was supported by the Ministry of Science and Technology, Ministry of Health and Welfare (MOHW109-TDU-B-211-134018) and National Health Research Institutes (NHRI-109A1-CACO-02202011).

\section{Authors' contributions}

Conception and study design: Y.M. Yeh, P.Y. Wu, K.F. Hsu, and M.R. Shen; Methodology: Y.M. Yeh and P.Y. Wu; Acquisition and interpretation of data: Y.M. Yeh, P.Y. Wu, P.C Lin, Y.T. Hsu, and J.Y. Chang; Statistical analysis: P.F. Su; Writing, review, and revision of the manuscript: Y.M. Yeh, P.Y. Wu, K.F. Hsu, and M.R. Shen; Administrative and material support: P.C Lin, Y.T Hsu and J.Y. Chang; Study supervision: M.R. Shen and K.F. Hsu

\section{Acknowledgments}

We thank the bioinformatics support from Kimforest LTD Taiwan and also appreciate the help of Dr. ChinHan Wu in animal studies.

\section{References}

1. Ferlay J, Soerjomataram I, Dikshit R, Eser S, Mathers C, Rebelo M, et al. Cancer incidence and mortality worldwide: sources, methods and major patterns in GLOBOCAN 2012. Int J Cancer. 2015;136:E359-86.

2. Lax SF, Kurman RJ. A dualistic model for endometrial carcinogenesis based on immunohistochemical and molecular genetic analyses. Verh Dtsch Ges Pathol. 1997;81:228-32.

3. Lewin SN, Herzog TJ, Barrena Medel NI, Deutsch I, Burke WM, Sun X, et al. Comparative performance of the 2009 international Federation of gynecology and obstetrics' staging system for uterine corpus cancer. Obstet Gynecol. 2010;116:1141-9.

4. National Comprehensive Cancer Network. Website https://www.nccn.org/.

5. Cancer Genome Atlas Research Network. Kandoth C, Schultz N, Cherniack AD, Akbani R, Liu Y, et al. Integrated genomic characterization of endometrial carcinoma. Nature. 2013; 497:67-73.

6. Aghajanian C, Filiaci V, Dizon DS, Carlson JW, Powell MA, Secord AA, et al. A phase II study of frontline paclitaxel/carboplatin/bevacizumab, paclitaxel/carboplatin/temsirolimus, or ixabepilone/carboplatin/bevacizumab in advanced/recurrent endometrial cancer. Gynecol Oncol. 2018;150:274-81. 
7. Hovelson DH, McDaniel AS, Cani AK, Johnson B, Rhodes K, Williams PD, et al. Development and validation of a scalable next-generation sequencing system for assessing relevant somatic variants in solid tumors. Neoplasia. 2015;17:385-99.

8. Aaron M, Matthew H, Eric B, Andrey S, Kristian C, Andrew K, et al. The Genome Analysis Toolkit: a MapReduce framework for analyzing next-generation DNA sequencing data. Genome Res. 2010;20:1297-303.

9. DePristo MA, Banks E, Poplin R, Garimella KV, Maguire JR, Hartl C, et al. A framework for variation discovery and genotyping using next-generation DNA sequencing data. Nat Genet. 2011;43:491-8.

10. Van der Auwera GA, Carneiro MO, Hartl C, Poplin R, Del Angel G, Levy-Moonshine A, et al. From FastQ data to high confidence variant calls: the Genome Analysis Toolkit best practices pipeline. Curr Protoc Bioinformatics. 2013;43:11.10.1-33.

11. Chen X, Schulz-Trieglaff O, Shaw R, Barnes B, Schlesinger F, Källberg M, et al. Manta: rapid detection of structural variants and indels for germline and cancer sequencing applications. Bioinformatics. 2016;32:1220-2.

12. Roller E, Ivakhno S, Lee S, Royce T, Tanner S. Canvas: versatile and scalable detection of copy number variants. Bioinformatics. 2016;32:2375-7.

13. McLaren W, Gil L, Hunt SE, Riat HS, Ritchie GR, Thormann A, et al. The ensembl variant effect predictor. Genome Biol. 2016;17:122.

14. Chen YF, Chen LH, Shen MR. The distinct role of STIM1 and STIM2 in the regulation of store-operated $\mathrm{Ca}^{2+}$ entry and cellular function. J Cell Physiol. 2019;234:8727-39.

15. Chen YF, Chiu WT, Chen YT, Lin PY, Huang HJ, Chou CY, et al. Calcium store sensor stromalinteraction molecule 1-dependent signaling plays an important role in cervical cancer growth, migration, and angiogenesis. Proc Natl Acad Sci USA. 2011;108:15225-30.

16. Gherardi E, Birchmeier W, Birchmeier C, Vande Woude G. Targeting MET in cancer: rationale and progress. Nat Rev Cancer. 2012;12:89-103.

17. Cortot AB, Kherrouche Z, Descarpentries C, Wislez M, Baldacci S, Furlan A, et al. Exon 14 deleted MET receptor as a new biomarker and target in cancers. J Natl Cancer Inst. 2017;109.

18. Goździk-Spychalska J, Szyszka-Barth K, Spychalski L, Ramlau K, Wójtowicz J, Batura-Gabryel H, et al. C-MET inhibitors in the treatment of lung cancer. Curr Treat Options Oncol. 2014;15:670-82.

19. Ye S, Li J, Hao K, Yan J, Zhou H. The efficacy and risk profile of c-Met inhibitors in non-small cell lung cancer: a meta-analysis. Sci Rep. 2016;6:35770.

20. Spassov VZ, Yan L. pH-selective mutagenesis of protein-protein interfaces: in silico design of therapeutic antibodies with prolonged half-life. Proteins. 2013;81:704-14.

21. Krishnaswamy S, Kanteti R, Duke-Cohan JS, Loganathan S, Liu W, Ma PC, et al. Ethnic differences and functional analysis of MET mutations in lung cancer. Clin Cancer Res. 2009;15:5714-23.

22. McAlpine J, Leon-Castillo A, Bosse T. The rise of a novel classification system for endometrial carcinoma; integration of molecular subclasses. J Pathol. 2018;244:538-49. 
23. Church DN, Stelloo E, Nout RA, Valtcheva N, Depreeuw J, Ter Haar N, et al. Prognostic significance of POLE proofreading mutations in endometrial cancer. J Natl Cancer Inst. 2015;107:402.

24. Sattler M, Reddy MM, Hasina R, Gangadhar T, Salgia R. The role of the c-Met pathway in lung cancer and the potential for targeted therapy. Adv Med Oncol. 2011;3:171-84.

25. Shieh JM, Tang YA, Yang TH, Chen CY, Hsu HS, Tan YH, et al. Lack of association of C-Met-N375S sequence variant with lung cancer susceptibility and prognosis. Int J Med Sci. 2013;10:988-94.

\section{Figures}
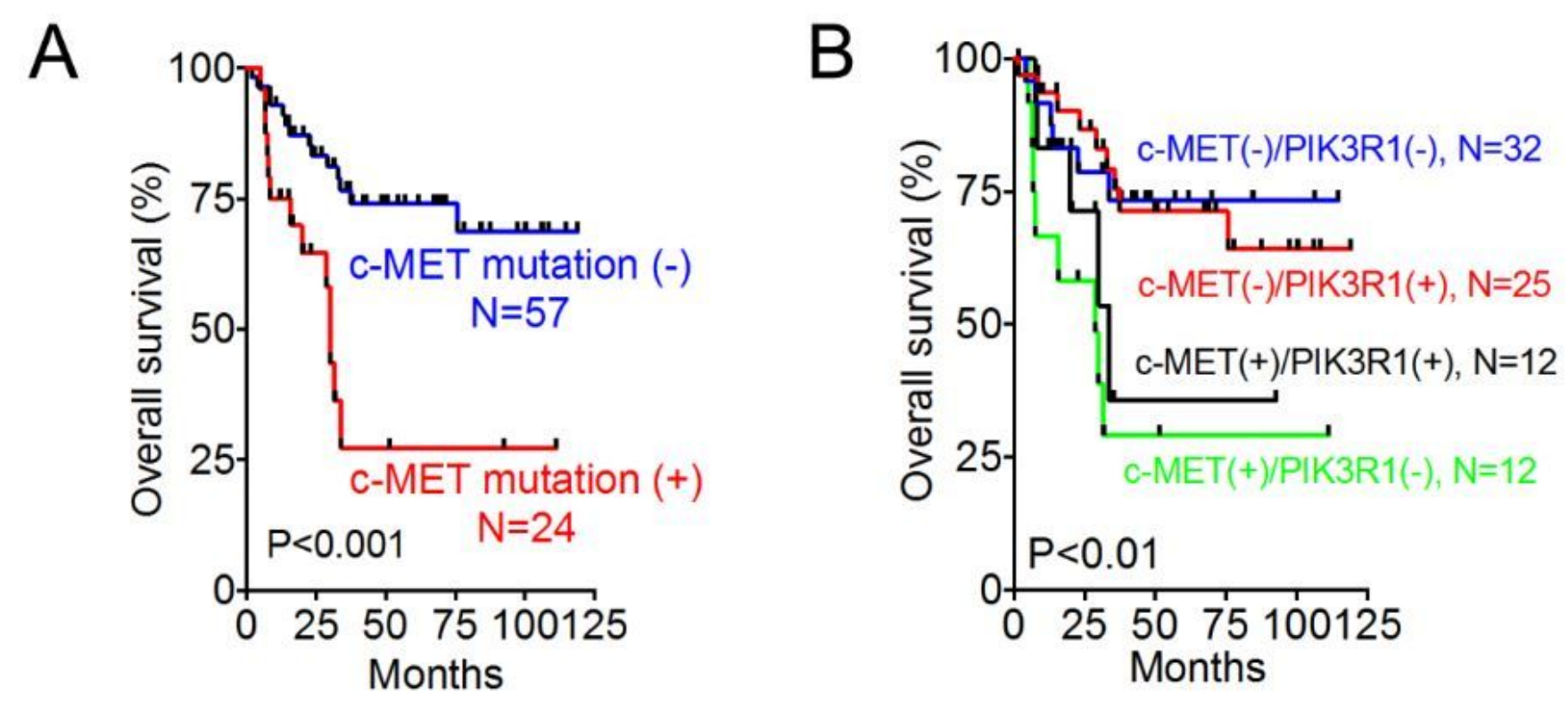

C
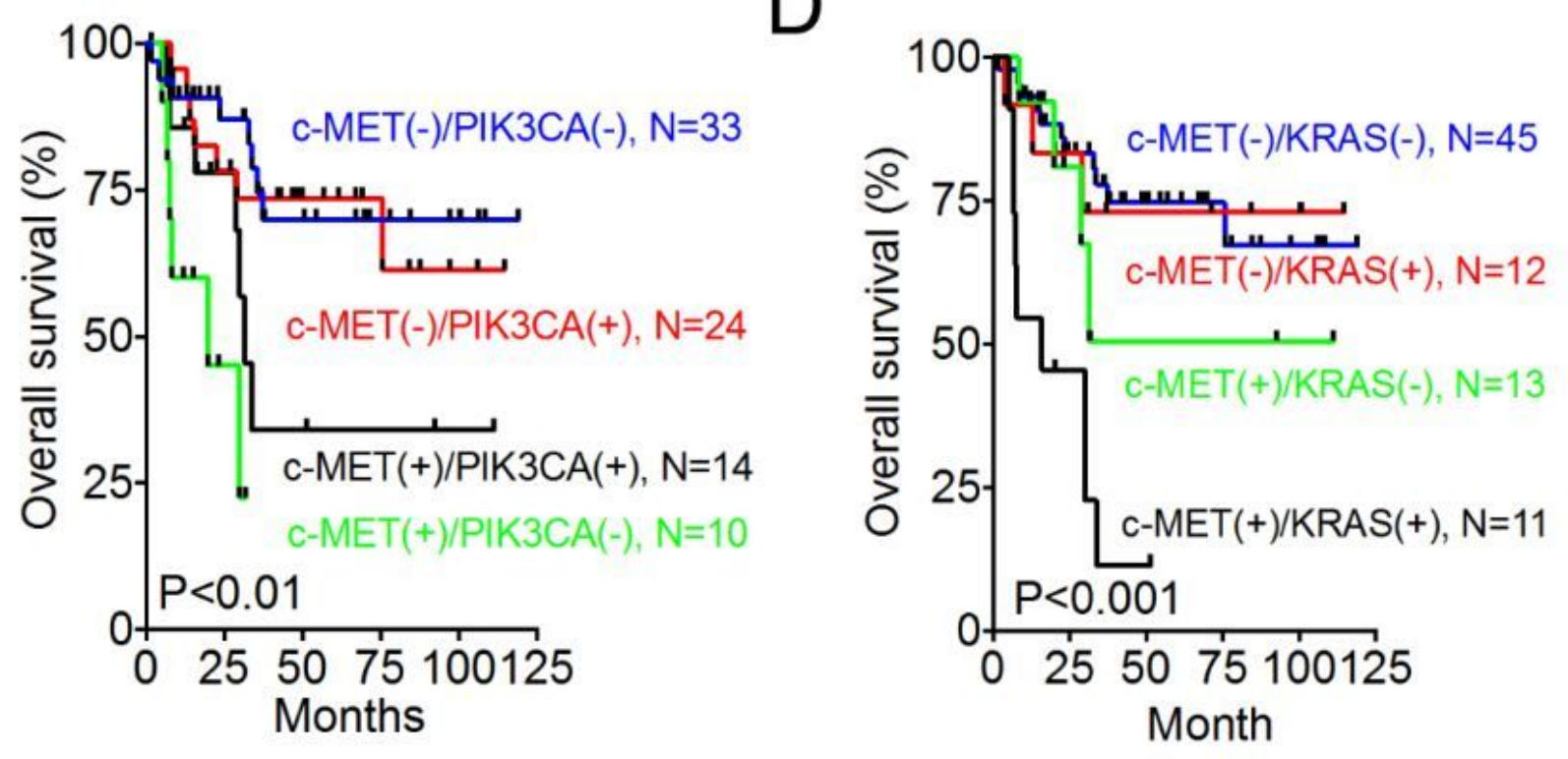

Figure 1 
Impact of MET mutation and concurrent genetic mutations on the clinical outcome of advanced endometrial cancer. (A) Kaplan-Meier curves for overall survival (OS) in patients with and without MET mutation compared using the log-rank test. (B D) Kaplan-Meier curves for OS were analyzed between the MET wild-type and MET mutant patients with or without concurrent PIK3R1 (B), PIK3CA mutation (C), and KRAS (D).

A

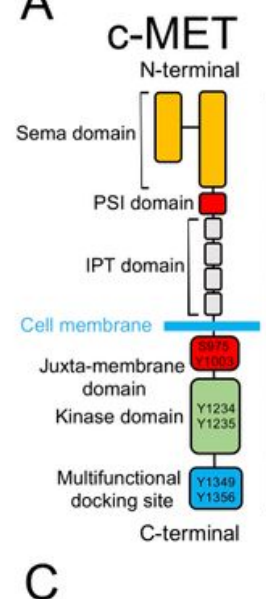

B

\begin{tabular}{|l|l|}
\hline $\begin{array}{c}\text { Associated } \\
\text { domain }\end{array}$ & \multicolumn{1}{c|}{$\begin{array}{c}\text { c-MET } \\
\text { mutation }\end{array}$} \\
\hline Sema & Exon $2(58.1 \%)$ \\
\hline IPT & Exon $10(1.6 \%)$ \\
\hline IPT & Exon $11(14.5 \%)$ \\
\hline $\begin{array}{l}\text { Juxta- } \\
\text { membrane }\end{array}$ & Exon $14(9.6 \%)$ \\
\hline Kinase & Exon $15(8.1 \%)$ \\
\hline Kinase & Exon $16(8.1 \%)$ \\
\hline
\end{tabular}

c-MET mutation

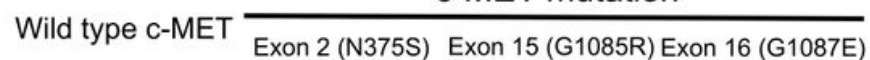
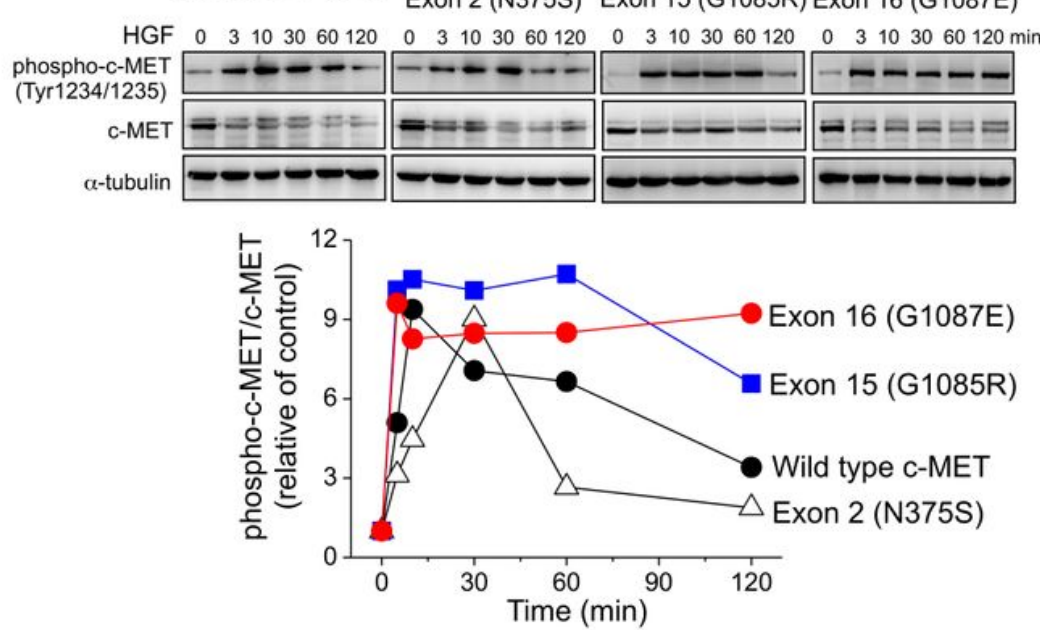

Fig 2

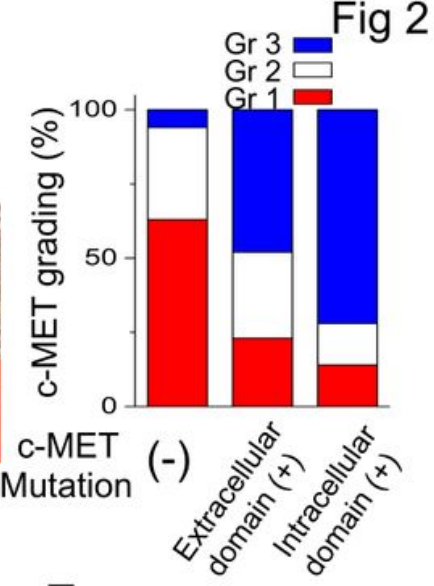

$\mathrm{E}$
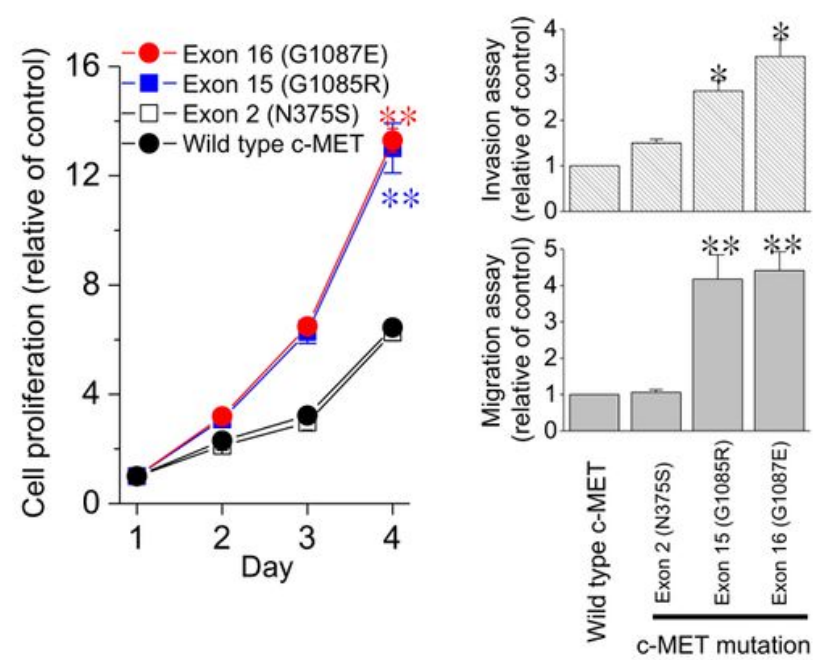

\section{Figure 2}

Differential effects of various MET mutations on c-MET phosphorylation and cellular functions. (A) The structure of C-MET (left) and distribution of MET mutations (right). Sema, semaphoring domain; PSI, plexin-semaphorin-integrin domain; IPT, immunoglobulin-plexin-transcription domain. (B) Representative images and grading of c-MET expression in endometrial cancer. The expression of c-MET was scored as grade $1-3$, according to the percentage of the positively-stained tumor cells and the intensity of the staining. (C) Time course of c-MET phosphorylation in response to HGF (50 ng/ mL) stimulation. Cell lysates from various clones of the endometrial cancer RL95-2 cells were immunoblotted with anti-c-MET, anti-phospho-c-MET, and anti-a-tubulin antibodies (upper). The expression levels were quantified and the ratios of phosphor-c-MET to total c-MET are shown (bottom). (D) Cell proliferation, invasion, and migration $(E)$ assays using various clones of the RL95-2 cells. Each value represents the mean \pm standard error of mean (SEM) from at least three experiments. ${ }^{*}, p<0.05 ; * *, p<0.01$. 
A
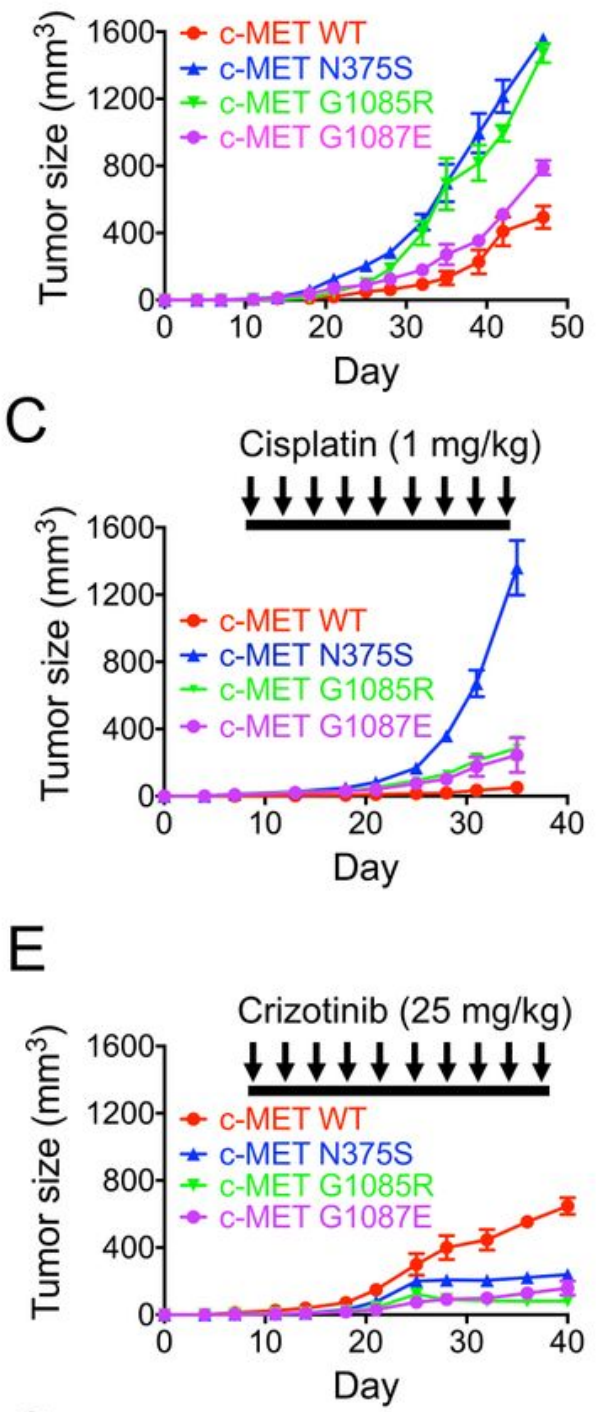

$G$

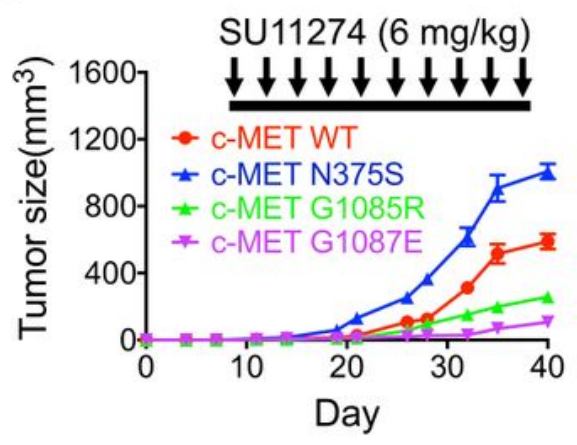

B

Fig 3

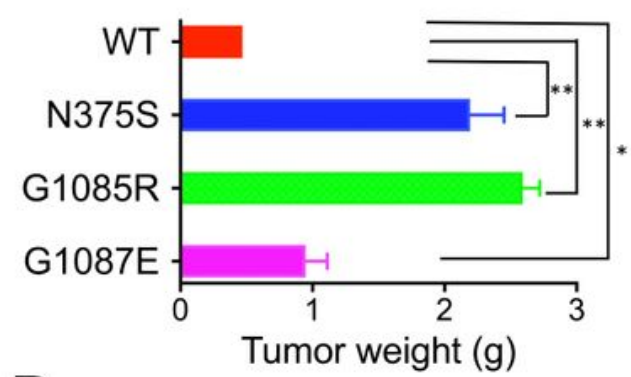

D

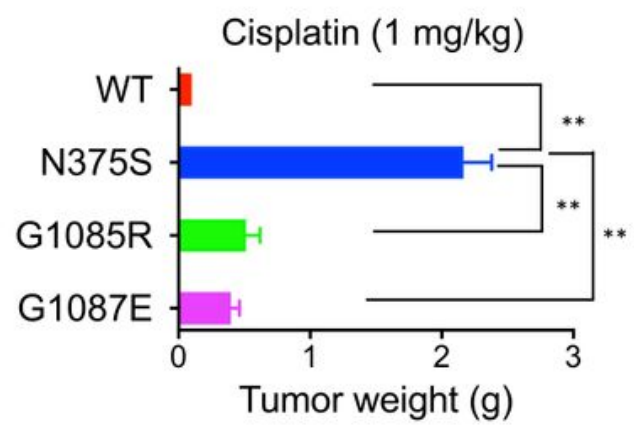

$\mathrm{F}$

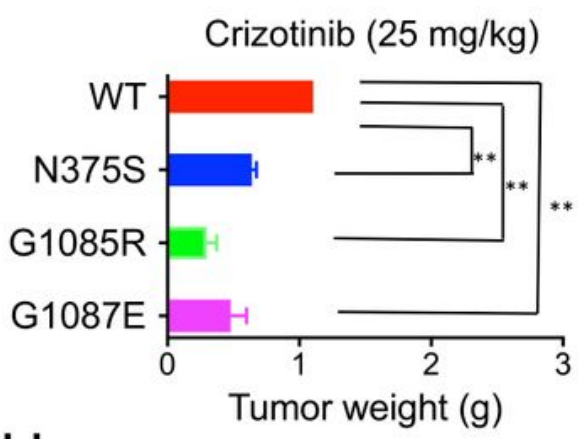

$\mathrm{H}$

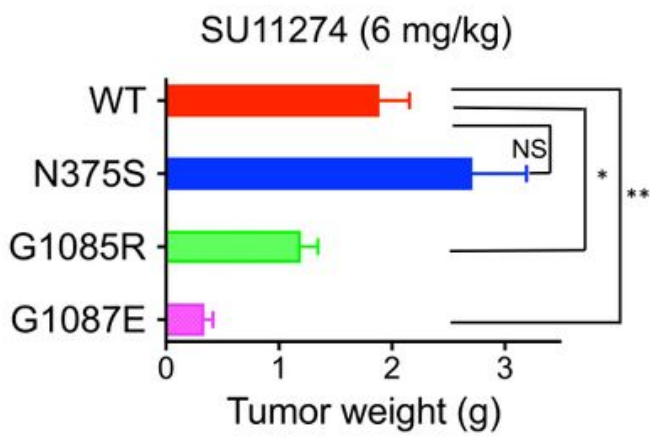

\section{Figure 3}

Antitumor effects of cisplatin and tyrosine kinase inhibitors in endometrial cancer xenografts harboring different MET mutations. 1 × 106 RL95-2 cells carrying wild-type or mutant MET, including N375S, G1085R, and G1087E, were injected into the posterior flank of SCID mice, subcutaneously. The tumors were allowed to grow and treated by observation only (A and B), cisplatin ( $1 \mathrm{mg} / \mathrm{kg}$ ) twice a week ( $\mathrm{C}$ and D), crizotinib (25 mg/ kg) by oral gavage twice a week (E and F), or SU11274 (6 mg/ kg) twice a week (G 
and $H)(n=5$ in each treatment group). Tumor size and body weight were measured twice a week. Data are presented as the mean tumor size \pm SEM. *, $p<0.05 ; * *, p<0.01 ; N S$, non-significance.

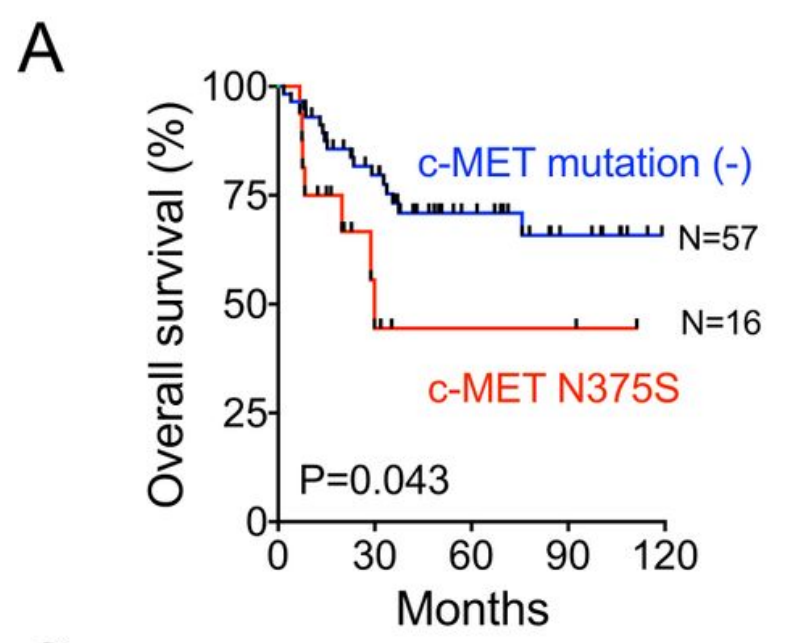

$\mathrm{B}$

Fig 4

\begin{tabular}{|c|c|c|}
\hline $\begin{array}{c}\text { C-MET } \\
\text { germline variants }\end{array}$ & $\begin{array}{c}\text { Taiwan } \\
\text { population (\%) }\end{array}$ & $\begin{array}{c}\text { Cancer } \\
\text { patients (\%) }\end{array}$ \\
\hline F206S & 0.4 & 0 \\
\hline L211W & 1 & 0 \\
\hline $\mathrm{D} 224 \mathrm{Y}$ & 0.2 & 0 \\
\hline L317P & 0.2 & 0 \\
\hline E355K & 0.2 & 0 \\
\hline N375S & 11.42 & 17.14 \\
\hline 1491T & 0.2 & 0 \\
\hline V546M & 0.2 & 0 \\
\hline V702L & 0.2 & 0 \\
\hline S805F & 0 & 2.86 \\
\hline L833F & 0 & 2.86 \\
\hline L900V & 0.2 & 0 \\
\hline A973V & 0 & 2.86 \\
\hline Q1067K & 0.2 & 0 \\
\hline $\mathrm{K} 1360 \mathrm{E}$ & 0.2 & 0 \\
\hline A1363T & 1.4 & 0 \\
\hline
\end{tabular}

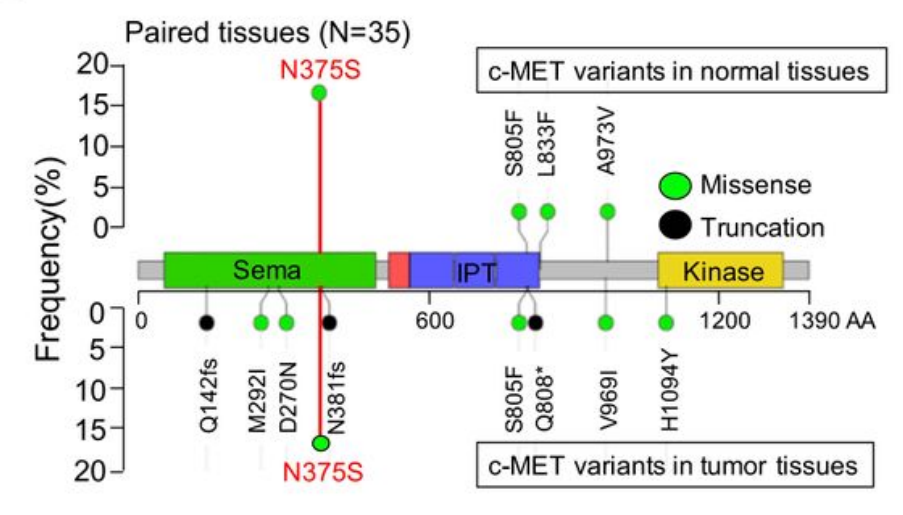

D

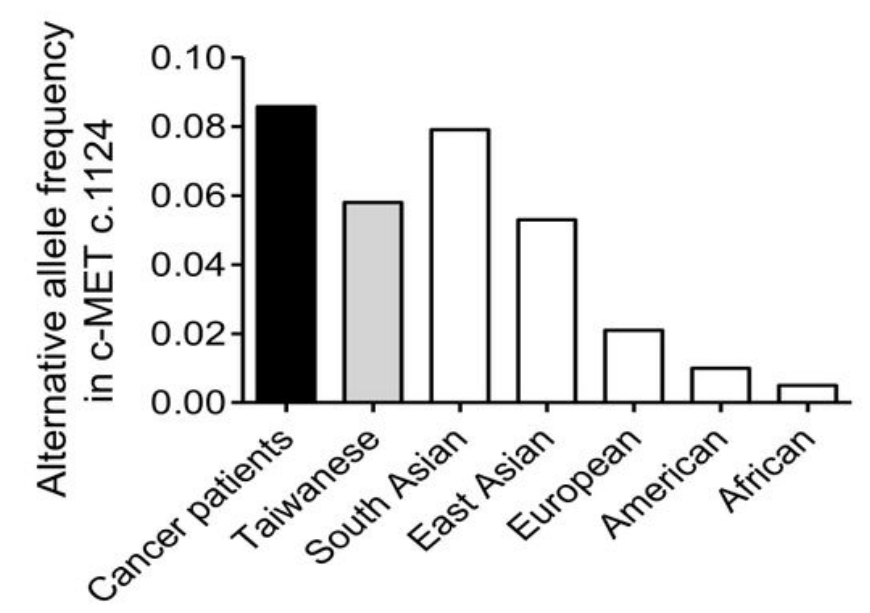

\section{Figure 4}

The clinical impact, distribution, and frequency of germline MET N375S variant. (A) OS of advanced endometrial cancer patients with and without the MET N375S variant. (B) The distribution and frequency of MET variants in 35 paired normal and tumor tissues. Whole-genome sequencing was used to analyze DNA extracted from blood samples or adjacent non-tumor specimens to determine germline MET variants. (C) The distribution and frequency of germline MET variants in cancer patients and the normal Taiwan population. The database from the Taiwan Biobank, which contained germline whole-genome sequencing data of 499 normal Taiwanese individuals, was used to compare the distribution and frequency of germline MET variants in the NCKUH cohort and normal Taiwan population. (D) The alternative allele frequency of MET c.1124 (N375S) in the different ethnic groups. The frequency of the variant allele in MET c.1124 was compared among the NCKUH cohort, normal Taiwanese population, and different ethnic groups enrolled in the 1,000 Genome Project.

\section{Supplementary Files}


This is a list of supplementary files associated with this preprint. Click to download.

- SupplementaryFigureS720200710.jpg

- SupplementaryFigureS620200710.jpg

- SupplementaryFigureS520200710.jpg

- SupplementaryFigureS4.jpg

- SupplementaryFigureS3.jpg

- SupplementaryFigureS2.jpg

- SupplementaryFigureS1.jpg

- SupplementaryTableS120200626.docx 